\title{
Real-time premature ventricular contractions detection based on Redundant Discrete Wavelet Transform
}

\author{
Ernano Arrais Junior ${ }^{1 *}$, Ricardo Alexsandro de Medeiros Valentim², Gláucio Bezerra Brandão² \\ ${ }^{1}$ Biomedical Signals Analysis Laboratory, Rural Federal University of Semi-Arid, Pau dos Ferros, RN, Brazil. \\ ${ }^{2}$ Department of Biomedical Engineering, Federal University of Rio Grande do Norte, Natal, RN, Brazil.
}

\begin{abstract}
Introduction: Premature Ventricular Contraction (PVC) is among the most common types of ventricular cardiac arrhythmia. However, it only poses danger if the person suffers from a heart disease, such as heart failure. Hence, this is an important factor to consider in heart disease people. This paper presents an ECG real-time analysis system for PVC detection. Methods: This system is based on threshold adaptive methods and Redundant Discrete Wavelet Transform (RDWT), with a real-time approach. This analysis is based on wavelet coefficients energy for PVC detection. It is presented also a study to find the most indicated wavelet mother for ECG analysis application among the following wavelet families: Daubechies, Coiflets and Symlets. The system detection performance was validated on the MIT-BIH Arrhythmia Database. Results: The best results were verified with db2 wavelet mother: the Sensitivity $\mathrm{Se}=99.18 \%$, Positive Predictive Value $\mathrm{P}+=99.15 \%$ and Specificity $\mathrm{Sp}=99.94 \%$, on 80.872 annotated beats, and $61.2 \mathrm{~s}$ processing speed for a half-hour record. Conclusion: The proposed system exhibits reliable PVC detection, with real-time approach, and a simple algorithmic structure that can be implemented in many platforms.
\end{abstract}

Keywords Electrocardiogram, Premature ventricular contraction, Redundant Discrete Wavelet Transform.

\section{Introduction}

Cardiovascular diseases are among the leading causes of death in the world. According to the World Health Organization (WHO), about $30 \%$ of these causes of death worldwide are related to cardiovascular problems. These numbers are closely linked to people's lifestyle and been increasing every year, generating greater concern on many health organizations (World..., 2017). More effective health actions are required, like prevention programs, diagnosis and treatment. In this sense, several technologies and methods have been developed to improve the health service in order to reduce the number of deaths and the costs involved with the healthcare system interventions (Braunwald et al., 2002; Valentim et al., 2015).

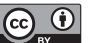

This is an Open Access article distributed under the terms of the Creative Commons Attribution License, which permits unrestricted use, distribution, and reproduction in any medium, provided the original work is properly cited.

How to cite this article: Arrais Junior E, Valentim RAM, Brandão GB. Real-time premature ventricular contractions detection based on Redundant Discrete Wavelet Transform. Res Biomed Eng. 2018; 34(3):187-197. DOI: 10.1590/2446-4740.01618.

*Corresponding author: Biomedical Signals Analysis Laboratory, Rural Federal University of Semi-Arid, BR 226, Km 405, Bairro São Geraldo, CEP 59900-000, Pau dos Ferros, RN, Brazil. E-mail: ernano_arrais@ieee.org

Received: 28 Febuary 2018 / Accepted: 14 June 2018
Currently, an alternative that have been improved in the primary healthcare system is the telecardiology, which enables patients telemonitoring, ensuring continuous vital signs monitoring, and provide greater mobility and comfort for patients (Chong et al., 2015; Gagnon et al., 2006; Hersh et al., 2001; Valentim et al., 2015). In this way, the automatic detection of cardiac signals (electrocardiogram - ECG) is important to cardiac disease diagnosis, and can be used like medical diagnosis assistance tool. However, the automatic ECG analyzing systems performance depends heavily upon the reliable and accurate detection of the ECG signal characteristic (QRS complex, as well as the $\mathrm{P}$ and $\mathrm{T}$ waves) (Dalvi et al., 2016; Gadêlha et al., 2012; Martis et al., 2014; Valentim et al., 2015).

In this work, an algorithm based on ECG signal with real-time approach has been developed in order to detect heart disease, in particular the Premature Ventricular Contraction (PVC) occurrence. The ECG reflects the heart electrical activity, it is a recording of the electric potential generated by electric behavior of the cardiac muscle tissue, the heart, and provides information about the heart state, enabling several noninvasive diagnostic of heart disease (Clifford et al., 2006; Malmivuo and Plonsey, 1995; Zago et al., 2015). Thus, a system that analyzes PVC is proposed, that is among the most common types of ventricular cardiac arrhythmia (Mann et al., 2014; Morris et al., 2009; Natale, 2007). 
The PVC is a cardiac rhythm abnormalities resulted from ectopic foci in the ventricles, and it is independent of the sinoatrial node pace set. It is frequently originated from the right ventricular outflow tract, but some can be originated from the left ventricular outflow tract, from aortic sinus cusp, from tricuspid and mitral valve annulus, and from coronary venous system, among other heart structures (Ge et al., 2012; Jia et al., 2011; Sayadi et al., 2010; Zheng et al., 2016).

The PVC generates one heartbeat that occurs abnormally early of the normal sinus rhythm, and usually it is not associated with $\mathrm{P}$ wave. This electrical event usually causes a break in the normal sinus heart activity - this break results from the gap between the PVC and the next normal heartbeat and is called compensatory pause (Malmivuo and Plonsey, 1995; Mann et al., 2014; Natale, 2007).

The PVC can occurs in person of any age and not necessarily represent cardiac risk, however it only pose danger if the person has any heart disease, such as heart failure. Thus, this is an important factor to consider in heart disease people. Recent studies indicate that the PVC can be an indicative of sudden cardiac death risk, and it is associated with mortality from acute myocardial infarction (Hirose et al., 2010; Inan et al., 2006; Iwasa et al., 2005; Lek-uthai et al., 2014). Therefore, the PVC detection has a particular interest for patients with heart disease treatment.

Hence, in the last years, several PVC detection system have been proposed for this issue: based on Artificial Neural Network (ANN) (Bortolan et al., 1991; Dalvi et al., 2016; Hu et al., 1997; Inan et al., 2006), Heuristic algorithm (Dotsinsky and Stoyanov, 2004), Bayesian framework (Sayadi et al., 2010), Support Vector Machine (SVM) (Shen et al., 2011), morphology ECG features (Chazal and Reilly, 2006; Chazal et al., 2004; Lek-uthai et al., 2014), Fuzzy Neural Network System (FNNS) (Lim, 2009), Wavelet Transform (Inan et al., 2006; Martis et al., 2013; Nazarahari et al., 2015; Orozco-Duque et al., 2013; Shyu et al., 2004; Yochum et al., 2016) and adaptive filter (Nieminaki et al., 1999; Solosenko et al., 2015). The main feature of most detection methods is a real-time analysis, however some methods have high mathematical complexity, which demands a high computational cost.

This paper presents a Wavelet-based algorithm for PVC detection, with a real-time approach. Adaptive threshold methods were used in parallel with the Wavelet Transform and the system was validated on MIT-BIH Arrhythmia Database (Moody and Mark, 2001; Goldberger et al., 2000). It was implemented the Redundant Discrete Wavelet Transform (RDWT), aiming a lower computational complexity.

\section{Methods}

\section{Wavelet transform}

The Wavelet Transform (WT) has been widely used in recent years in various areas and several applications, such as power system quality (Andrade and Leao, 2014; Arrais et al., 2014; Costa, 2014), signal processing (Pedireddi and Srinivasan, 2010; Saleh et al., 2012), biomedical engineering (Arrais et al., 2016; Dalvi et al., 2016; Di Marco and Chiari, 2011; Kim et al., 2011; Madeiro et al., 2009), among others. WT provides a compact and flexible analysis in time and frequency domain, allowing different resolution levels, besides presenting various base functions (Wavelet families), which allow a more specific analysis in order to adapt the analysis of signal behavior and so achieve the best possible results (Burrus et al., 1997; Mallat, 1989, 2008; Percival and Walden, 2000).

WT allows multiresolution analysis, such that a discrete signal can be decomposed and analyzed at different resolution levels (or scales) (Burrus et al., 1997; Mallat, 1989, 2008). Thus, a discretized signal can be decomposed in terms of wavelet coefficients and scaling coefficients at various levels of resolution through a digital filtering process. This filtering process divides the input signal into two frequency bands, high frequency signals and low frequency signals. The wavelet coefficients is the high-pass filters results and the scaling coefficients is the low-pass filters results (Burrus et al., 1997; Mallat, 1989, 2008; Percival and Walden, 2000).

Wavelet Transforms can be used for continuous time (Continuous Wavelet Transform - CWT) or discrete time (Discrete Wavelet Transform - DWT) signals applications. In relation to basic functions there are several Wavelet families as Morlet, Biorthogonal, Mexican Hat, Harr, Daubechies, Meyer, Coiflets, Symlets, and many others, both in the Real domain and Complex domain numbers (Daubechies, 1992; Grossmann and Morlet, 1984; Mallat, 2008; Percival and Walden, 2000).

For cardiac signal analysis the Wavelet family most used is the Daubechies, especially the mother Wavelet db4 (Arrais et al., 2016; Sasikala and Wahidabanu, 2010; Singh and Tiwari, 2006). However, the ECG signal analysis with $\mathrm{db} 4$ explanation is not clear in this works. In this paper is used the Redundant Discrete Wavelet Transform (RDWT), a DWT variant version, and is compared the system performance with some mother wavelets from Daubechies, Coiflets and Symlets Wavelet families in order to find the best mother wavelet for ECG signals analysis application.

\section{Redundant discrete wavelet transform}

RDWT is a DWT variant version, and presents the basic characteristics of it: the original signal is decomposed into two components, wavelet and scaling coefficients, and the filter coefficients for the calculation of each 
component are related, the high-pass and low-pass filters, respectively (Daubechies, 1992; Grossmann and Morlet, 1984; Mallat, 2008; Percival and Walden, 2000). Although the DWT provides relatively simple implementation complexity, speed and low computational effort, the RDWT has become interesting due to the fact that DWT present some limitations for some specific applications such as the detection of the QRS complex in real-time analysis (Arrais et al., 2016).

The major difference between the DWT and RDWT is the subsampling process: the DWT presents time variant behavior due to the subsampling process used to calculate the wavelet and scaling coefficients, whereas the RDWT not present the subsampling process, so this mathematical tool provides time invariant behavior, resulting in less significant delay over the signal reconstruction process if compared to DWT (Arrais et al., 2016; Percival and Walden, 2000). In general, the main differences are listed below:

- RDWT can be applied to any number of samples, whereas DWT requires is a power of two number sample due to the subsampling process by two;

- DWT is an orthonormal transformation, whereas RDWT is a non orthogonal transformation;

- RDWT is an invariant time transform, since it does not perform subsampling process by two in the signal sample, whereas DWT performs the subsampling process by two, resulting in a time variant behavior.

The RDWT mathematical model for real-time approach is given by the following equations (Arrais et al., 2014; Costa and Driesen, 2013): The Equation 1 provides the scaling coefficients, $s_{j}$, and the Equation 2 provides the wavelet coefficients, $\omega_{j}$, both in the level resolution (scale) $j$, obtained by convolution of the scaling coefficients $s_{j-1}$ by $\tilde{g}$ and $\tilde{h}$ filters, of the scale $j-1$. The $\tilde{g}$ is the low-pass filter coefficients and $\tilde{h}$ is the high-pass filter coefficients for calculating the scaling and wavelet coefficients, respectively.

$$
\begin{aligned}
& s_{j}(k)=\sum_{l=1}^{L-1} \tilde{g}(l) s_{j-1}(k+l-L) \\
& \omega_{j}(k)=\sum_{l=1}^{L-1} \tilde{h}(l) s_{j-1}(k+l-L)
\end{aligned}
$$

since $k \geq L-1: k$ is the current sampling in the real-time analysis and $L$ is the length of the filters. The variable $l$ is incremental variable of summation.

Figure 1 shows a decomposition process block diagram for RDWT of a signal $x_{0}\left(s_{0}\right)$ with sampling frequency $(f)$ in two resolution level (first and second scale), that provides the scaling $\left(s_{1}\right.$ and $s_{2}$ ) and wavelet $\left(\omega_{1}\right.$ and $\left.\omega_{2}\right)$ coefficients. This decomposition process

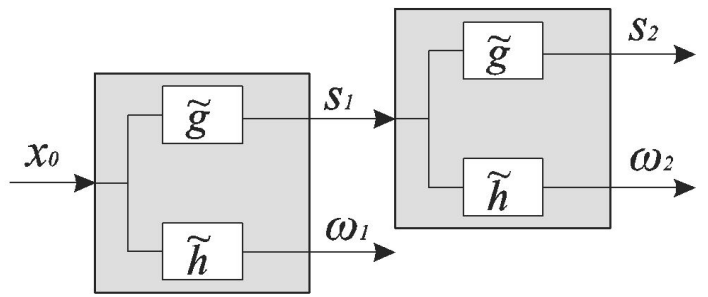

Figure 1. Block diagram illustrating the RDWT decomposition process in two resolutions levels (or two resolutions scales). $x_{0}$ is the original signal, $s_{1}$ and $s_{2}$ are the scaling coefficients on the first and second resolutions levels, respectively; $\omega_{l}$ and $\omega_{2}$ are the wavelet coefficients in the first and second resolutions levels, respectively; $\tilde{g}$ and $\tilde{h}$ are the low-pass and high-pass filters coefficients, respectively.

is based on the pyramidal algorithm of Mallat (Mallat, 1989, 2008). The filters coefficients $\tilde{g}$ and $\tilde{h}$ are obtained based on the DWT filters ( $g$ and $h$ ) (Costa and Driesen, 2013; Percival and Walden, 2000), as defined below (Equations 3 and 4):

$$
\tilde{g}(l)=g(l) / \sqrt{2}
$$

$\tilde{h}(l)=h(l) / \sqrt{2}$

The signal energy can be calculated based on the scaling and wavelet coefficients: in according to Parseval's theorem, the signal energy can be partitioned in terms of the wavelet and scaling coefficients energy (Burrus et al., 1997). In other words, the energy of the original signal $x_{0}$, with $k_{t}$ sample, is equal to the sum of the wavelet coefficients energies of all resolution levels, $1 \leq j \leq J$, with the scaling coefficients energy of the last resolution level, $J$, with $J \leq J_{\max }$ (Burrus et al., 1997; Costa and Driesen, 2013). The energy signal can be calculated by Equation 5:

$$
\sum_{k=1}^{k_{t}}|x(k)|^{2}=\sum_{k=1}^{k_{t}}\left|s_{J}(k)\right|^{2}+\sum_{j=1}^{J} \sum_{k=1}^{k_{t}}\left|\omega_{j}(k)\right|^{2}
$$

where:

- $\sum_{k=1}^{k_{t}}|x(k)|^{2}-$ It is the energy of the original signal $x_{0}$,

- $\sum_{k=1}^{k_{t}}\left|s_{J}(k)\right|^{2}-$ It is the energy of the scaling coefficients at level $J$,

- $\sum_{k=1}^{k_{t}}\left|\omega_{j}(k)\right|^{2}-$ It is the energy of the wavelet coefficients at level $j$.

\section{Benchmark databases}

Several standard ECG databases are available for the evaluation of heart disease detection algorithms (Kohler et al., 2002; Martis et al., 2014). The algorithm performance on a standard database is not the main parameter to propose to apply in a clinical environment, 
but it provides a good performance comparison between algorithm detections. In this research work was used the MIT/BIH arrhythmia database, since it is one of the most used to evaluate the algorithm ECG detections (Arrais et al., 2016; Hamilton and Tompkins, 1986; Li et al., 1995; Martinez et al., 2004; Pan and Tompkins, 1985).

This database is obtained over 4,000 long-term Holter recordings at Beth Israel Hospital Arrhythmia Laboratory between the years of 1975 and 1979 from patients under different cardiac arrhythmias. It contains 48.5 hours of recordings with two-channel and sampling rate of $360 \mathrm{~Hz}$. However, only one of the ECG channels was used (channel 1 - a modified limb lead II (MLII)). However, the database contains records of modified leads V1, V2 and V5, and, in one instance, also V4. The subject's records were obtained from 25 men aged 32 to 89 years, and 22 women aged 23 to 89 years (Goldberger et al., 2000; Moody and Mark, 2001). It was analyzed 36 recordings, although the database contains 48 recordings, by reason of external noise in some recordings that disturbed the ECG signal behavior.

\section{System description}

The system for PVC detection is based on the RDWT and adaptive threshold methods. It was described on Matlab 2014a platform version. The Figure 2 shows the system description flowchart. The RDWT provides two coefficients, the scaling and the wavelet coefficients, and the algorithm proposed is based on the energy wavelet coefficients for PVC detection. The ECG features is obtained with the RDWT and the detection process is through adaptive threshold methods based on mean value ECG signal energy on all resolution levels, therefore there is one threshold for each level.

In the proposed system, each ECG signal sample (data record from MIT-BIH databases) is analyzed in real-time approach. The RDWT-based energy wavelet coefficients are analyzed to detect the PVC. Each energy wavelet coefficient sample is compared with a threshold value, and if it is higher than the threshold value, the sample is a possible PVC occurrence indicative. Hence, this sample is stored and will be compared with the next sample (remaining). Therefore, these selected samples are compared and the one that has the highest value (amplitude peak) corresponds to the energy maximum value and a PVC occurrence.

The energy PVC value is much higher than the normal ECG energy module value. For this reason that the PVC can be detect based on energy signal. The variable $e[n]$ is the $n$ sample energy wavelet coefficients, provided by RDWT. It is used an auxiliary variable $E_{p_{-} a u x}$ to store a temporary energy sample value. The threshold value $t h r$ is obtained from empirical way, according to computed test, and it is used to detect the possible PVC occurrence.

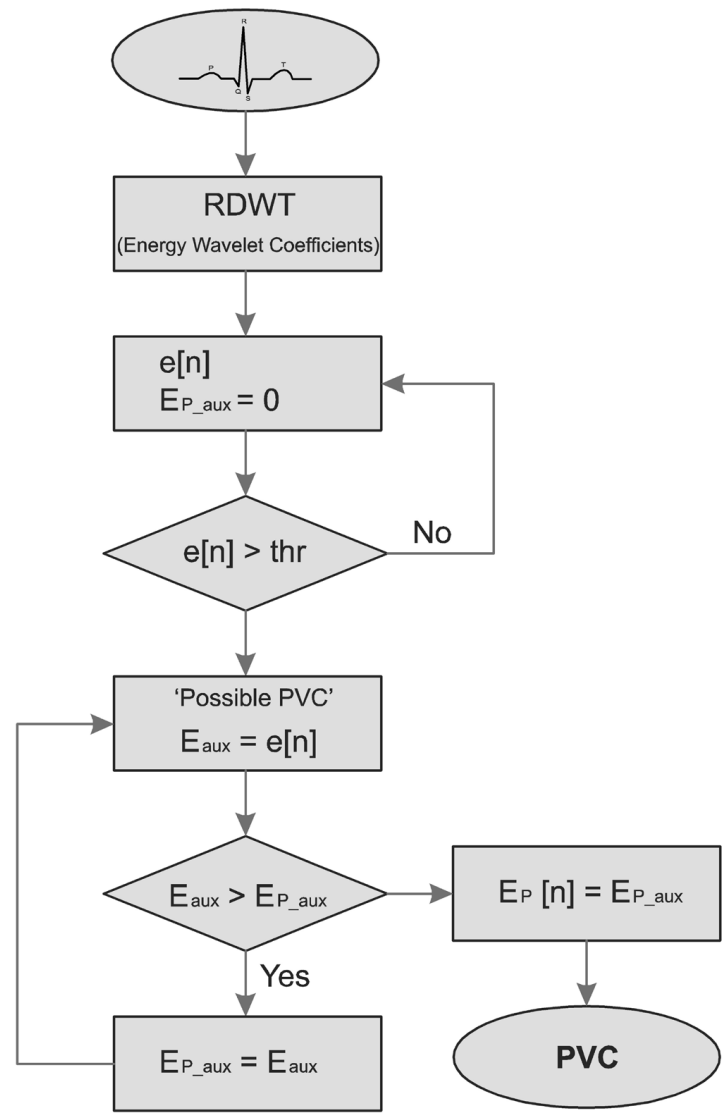

Figure 2. System flowchart. The energy wavelet coefficients algorithm analysis is based on RDWT. The variable $e[n]$ is the $n$ sample energy wavelet coefficients. $E_{p \text { aux }}$ is an auxiliary variable used to store a temporary energy value sample. The $t h r$ is the threshold value used to detect the possible PVC occurrence. If the energy wavelet coefficient sample $(e[n])$ is higher than the threshold $(t h r)$, this sample is stored in the auxiliary variable $E_{a u x}$. So, the algorithm compares each value $E_{a u x}$ with the next sample. Then, the current sample is stored in $E_{a u x}$ and the previous sample in the $E_{p a x}$. When the current sample $\left(E_{a u x}\right)$ is less than the previous sample $\left(\bar{E}_{p a x x}\right)$, this sample is stored in a vector $E_{p}[n]$, and this indicates possible PVC. Thus, the ECG signal is analyzed and all indicative PVC occurrence is stored in a vector. This algorithm is computed for all scales, and each scale has one associated threshold value. Thus, the PVC occurrence can be detected in all scales, although the three last levels are predominant.

The threshold value is updated in according to the mean energy value in each minute ECG record.

If the energy wavelet coefficient sample $(e[n])$ is greater than the threshold $(t h r)$, this sample is stored in the auxiliary variable $E_{a u x}$. Then, the algorithm compares each value $E_{\text {aux }}$ with the next sample. Then, the current sample is stored in $E_{a u x}$ and the last sample in the $E_{p_{-} a u x}$. When the current sample (Eaux) is less than the previous sample (Epaux), this sample is stored in a vector $E_{p}[n]$, and this indicates possible PVC. Thus, the ECG signal is analyzed and all indicative PVC occurrences are stored in a vector. 
In this work the RDWT was implemented with six resolution levels (or scale). The sample frequency used was $350 \mathrm{~Hz}$, in order to optimize the distribution of the ECG signal energy into resolution levels, to facilitate the signal analysis, according to ECG power spectra (Thakor et al., 1984). The Table 1 presents the frequency bands for each RDWT resolution level of the wavelet coefficients.

\section{Results}

In this paper it was analyzed the effect of the wavelet families Daubechies, Coiflets and Symlets, in order to find the best wavelet for ECG proposed analysis. This analyze motivation by virtue of many works use the mother wavelet db4 (Daubechies family), but the reason is not clear. The Table 2 shows the comparative results, based on standard deviation analysis between the ECG energy signal and the energy scaling coefficients at all resolution level. It was analyzed the following mother wavelets: Daubechies (db2, db4, db6, db8 and db12), Coiflets (coif6, coif12 and coif18) and Symlets (sym4, sym6, sym8 and sym10).

The energy coefficients standard deviation provides a good dispersion measure to verify the energy behavior ECG signal analyzed by the pyramidal algorithm, since the energy loss in the detection process will result in low performance. This analysis was computed for all

Table 1. Frequency bands for each resolution level (scale) of RDWT for the Wavelet coefficients.

\begin{tabular}{cc}
\hline S & Frequency range $[\mathrm{Hz}]$ \\
\hline 1 & $87.5-175$ \\
2 & $43.75-87.5$ \\
3 & $21.875-43.75$ \\
4 & $10.9375-21.875$ \\
5 & $5.4688-10.9375$ \\
6 & $2.7344-5.4688$ \\
\hline
\end{tabular}

resolution level in order to find the lowest variation of the standard deviation between the levels.

Based on the results the lowest mother wavelet filter order provides the best standard deviation between the energy original signal and the wavelet coefficients energy for each level. So, the reliability and effectiveness of cardiac signal analysis algorithms based on Wavelet Transform are closely linked to the wavelet filters order used, with better results for low-order filters. Thereby, due to this comparative study, it was chosen the Daubechies Wavelet family for signal analysis.

Although the study indicates that $\mathrm{db} 2$ is the best for ECG application, in this paper the mother Wavelets $\mathrm{db} 2, \mathrm{db} 4$, coif6 and sym 4 were verified, in order to ascertain which provides the best performance, since these mother wavelets provided the lowest standard deviation value for their respective wavelet families. This motivation is also due to the large amount of works that use db4 for ECG analysis and the search for a faster algorithm.

The system was validated on MIT-BIH Arrhythmia Database (Goldberger et al., 2000; Moody and Mark, 2001), and the detection algorithms performance is evaluated according to three parameters, Sensitivity (Se), Positive Predictivity $(\mathrm{P}+)$ and Specificity $(\mathrm{Sp})$ (Kohler et al., 2002; Wang, 2013):

$$
S_{e}=\frac{T P}{T P+F N} \times 100
$$

$$
\begin{aligned}
& P^{+}=\frac{T P}{T P+F P} \times 100 \\
& S p=\frac{T N}{T P+F N} \times 100
\end{aligned}
$$

where $T P$ is the True Positive, the $F N$ is the False Negative, $F P$ the False Positive and $T N$ the True Negative values.

Table 2. Comparative study among three Wavelet families for ECG signal analyses. Normalized standard deviation analysis between the ECG energy signal and the energy scaling coefficients at all resolution level. It was calculated for three Wavelet families and some of their mothers wavelets: Daubechies (db2, db4 db6, db8 and db12), Coiflets (coif6, coif12 and coif18) and Symlets (sym4, sym6, sym8 and sym10). It was computed the

\begin{tabular}{|c|c|c|c|c|c|c|c|c|c|c|c|c|}
\hline \multirow{2}{*}{ Scale } & \multicolumn{5}{|c|}{ Daubechies } & \multicolumn{3}{|c|}{ Coiflets } & \multicolumn{4}{|c|}{ Symlets } \\
\hline & db2 & db4 & db6 & db8 & db12 & coif6 & coif12 & coif18 & sym4 & sym6 & sym8 & sym 10 \\
\hline 1 & 0.911 & 0.972 & 0.990 & 0.997 & 0.998 & 0.998 & 0.973 & 0.997 & 0.992 & 0.972 & 0.990 & 0.997 \\
\hline 2 & 1.000 & 0.410 & 0.298 & 0.271 & 0.269 & 0.279 & 0.424 & 0.354 & 0.470 & 0.410 & 0.298 & 0.269 \\
\hline 3 & 1.000 & 0.729 & 0.651 & 0.615 & 0.594 & 0.581 & 0.721 & 0.571 & 0.547 & 0.729 & 0.651 & 0.616 \\
\hline 4 & 1.000 & 0.926 & 0.904 & 0.894 & 0.888 & 0.883 & 0.923 & 0.881 & 0.872 & 0.926 & 0.904 & 0.894 \\
\hline 5 & 1.000 & 0.900 & 0.875 & 0.865 & 0.860 & 0.857 & 0.897 & 0.854 & 0.847 & 0.900 & 0.875 & 0.865 \\
\hline 6 & 0.905 & 0.970 & 0.989 & 0.997 & 0.999 & 0.998 & 0.972 & 0.997 & 0.992 & 0.970 & 0.989 & 0.997 \\
\hline
\end{tabular}
following recordings: $100,101,102,103,104,105,106,107,108,109,111,112,113,114,115,116,117,118,119,121,122,123,124,200,201$, $202,203,205,207$ and 208. 
Figure 3 shows the algorithmic results based on $\mathrm{db} 2$ mother wavelet for the record 100 analysis from MIT-BIH Arrhythmia Database. Figure 3a is a range time where occurs a PVC. Figure $3 b$ presents the wavelet coefficients for this range time. It can be observed that larger variations are observed on the scales $3\left(\omega_{3}\right)$ and $4\left(\omega_{4}\right)$, but during the PVC a larger variations are observed on the scales $4\left(\omega_{4}\right)$, $5\left(\omega_{5}\right)$ and $6\left(\omega_{6}\right)$, principally the coefficients in the scale $5\left(\omega_{5}\right)$. It can be verified that when a PVC occurs the wavelet coefficients increase, if compared to normal heartbeat wavelet coefficients.

The largest variation observed on wavelet coefficients at scale $5\left(\omega_{5}\right)$ implies that the highest energy portion is on the scale 5 . Figure $3 \mathrm{c}$ illustrates the wavelet coefficients energy on the six resolutions levels. It can be verified that the energy increases enough when the PVC occurs, and this event can indicate a PVC occurrence. The energies values increase in all resolution levels, but in last three levels are most significant, in specially at level 5 (wavelet coefficients energy $\omega_{5}$ ), and associated with threshold methods can detect the PVC occurrence.

Figure 4 shows the algorithm results based on $\mathrm{db} 2$ mother wavelet for the record 102 analysis (MIT-BIH Arrhythmia Database). In this case, there is a change in the ECG signal behavior after the occurrence of the PVC due to the action of a pacemaker (Figure 4a), to control the heart rate. Figure $4 \mathrm{~b}$ presents the wavelet coefficients energy on all scales $(\omega 1, \omega 2, \omega 3, \omega 4, \omega 5$ and $\omega 6)$. If compared to Figure 3 , the highest energy portion is concentrated in the $6^{\text {th }}$ scale, but the energies are also most significant on last two scale. So the detection can occur in any scale or more than one, and the threshold technical provides good comparative analysis for all resolution level.

Table 3 presents the algorithm comparative results on the following mother wavelets: db2, db4, coif6 and sym4. The table shows the Sensitivity (Se), Positive Predictivity $(\mathrm{P}+)$ and Specificity $(\mathrm{Sp})$ parameters used for PVC detection performance comparison.

Table 4 presents the system analysis based on $\mathrm{db} 2$ for 36 records from MIT-BIH Arrhythmia Database, on 80.872 annotated beats, being 3.408 annotated PVC beats. The table shows besides the PVC detected for each data record, the True Positive (TP) and True Negative (TN) value, the False Positive (FP) and False Negative (FN) value, and amount of failures.

Table 5 lists the processing speed analysis for each analyzed mother wavelet ( $\mathrm{db} 2, \mathrm{db} 4$, coif6 and sym4) taking into account a half-hour record from MIT-BIH
Arrhythmia Database. The processing time is computed in seconds.

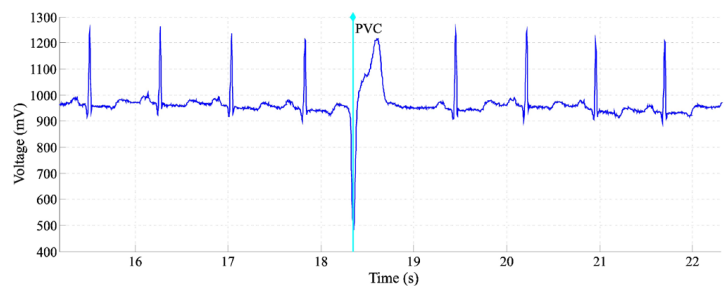

(a)

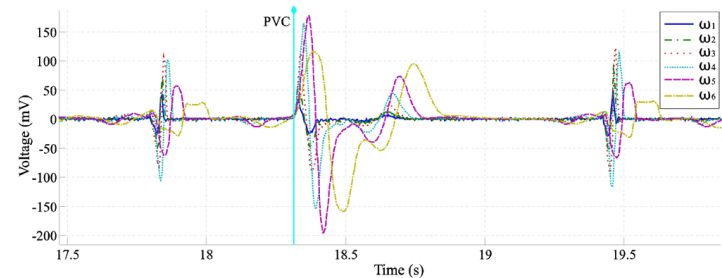

(b)

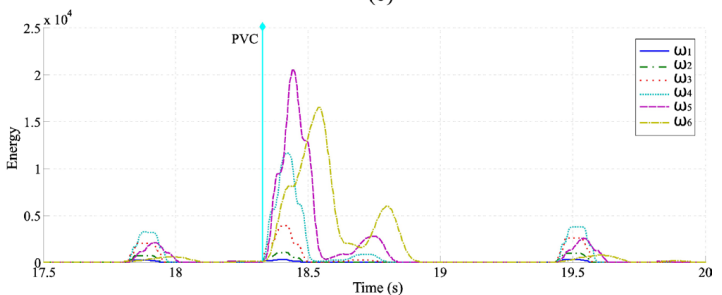

(c)

Figure 3. Algorithm results based on $\mathrm{db} 2$ mother wavelet for record 100 analysis: (a) is the ECG signal interval that occurs a PVC; (b) is the wavelet coefficients in the six resolution levels $(\omega 1, \omega 2, \omega 3, \omega 4, \omega 5$ and $\omega 6)$; (c) wavelet coefficients energy on all scales.

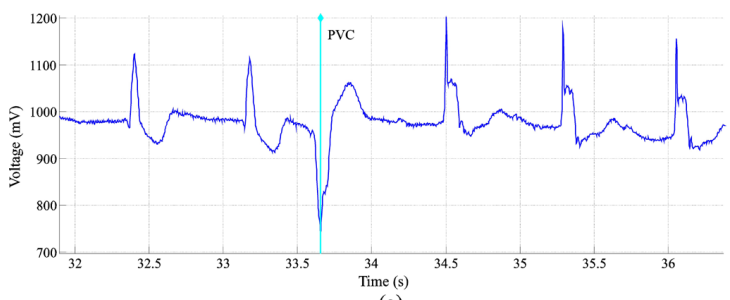

(a)

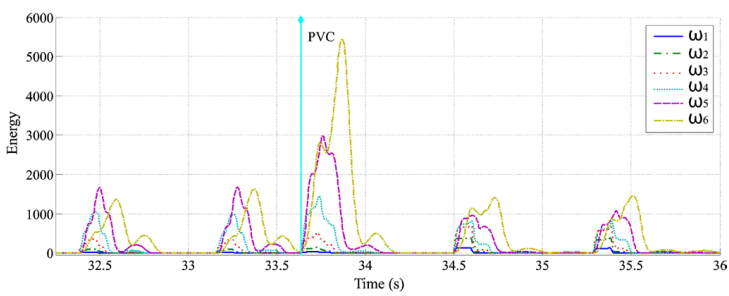

(b)

Figure 4. Algorithm results based on $\mathrm{db} 2$ for record 102 analysis: (a) is the ECG signal interval that present a PVC occurrence; (b) presents the wavelet coefficients energy in the six resolution levels $(\omega 1, \omega 2, \omega 3$, $\omega 4, \omega 5$ and $\omega 6)$. 
Table 3. PVC detection performance comparison on several mother wavelets: $\mathrm{db} 2, \mathrm{db} 4$, coif6 and sym 4 . Where $\mathrm{Se}$ is the Sensitivity value, $\mathrm{P}^{+}$the Positive Predictivity value and $S p$ the Specificity value.

\begin{tabular}{cccc}
\hline Mother Wavelet & $\boldsymbol{S e}(\boldsymbol{\%})$ & $\mathbf{P}^{+}(\mathbf{\%})$ & Sp $(\mathbf{\%})$ \\
\hline db2 & 99.18 & 99.15 & 99.94 \\
db4 & 99.23 & 99.09 & 99.96 \\
coif6 & 97.12 & 96.20 & 99.70 \\
sym4 & 99.23 & 99.09 & 99.96 \\
\hline
\end{tabular}

Table 4. PVC detection performance on the MIT-BIH Arrhythmia Database for db2 mother wavelet: each data record number represents the respective record on the database. The column Total Beats show the annotated beats number in each data record. The Total PVC column indicates the number PVC occurrence in the each data record. The TP and $T N$ are the True Positive and True Negative value, respectively. The $F P$ is the False Positive value and $F N$ is the False Negative value. The columns Failed Detection shows the error detection algorithm in absolute and perceptual value, respectively.

\begin{tabular}{|c|c|c|c|c|c|c|c|c|}
\hline $\begin{array}{l}\text { Data } \\
\text { record }\end{array}$ & Total beats & $\begin{array}{l}\text { Total } \\
\text { PVC }\end{array}$ & TP & $\mathbf{T N}$ & FP & FN & $\begin{array}{c}\text { Failed } \\
\text { detection }\end{array}$ & $\begin{array}{c}\text { Failed } \\
\text { detection } \\
(\%)\end{array}$ \\
\hline 100 & 2273 & 1 & 1 & 2272 & 0 & 0 & 0 & 0 \\
\hline 101 & 1865 & 1 & 1 & 1864 & 0 & 0 & 0 & 0 \\
\hline 102 & 2187 & 4 & 4 & 2183 & 0 & 0 & 0 & 0 \\
\hline 103 & 2084 & 0 & 0 & 2084 & 0 & 0 & 0 & 0 \\
\hline 105 & 2572 & 41 & 36 & 2526 & 0 & 5 & 5 & 12.20 \\
\hline 106 & 2027 & 520 & 520 & 1507 & 0 & 0 & 0 & 0 \\
\hline 107 & 2137 & 59 & 69 & 2068 & 8 & 2 & 10 & 16.95 \\
\hline 108 & 1763 & 17 & 17 & 1746 & 0 & 0 & 0 & 0 \\
\hline 109 & 2532 & 38 & 38 & 2494 & 0 & 0 & 0 & 0 \\
\hline 111 & 2124 & 1 & 1 & 2123 & 0 & 0 & 0 & 0 \\
\hline 112 & 2539 & 0 & 0 & 2539 & 0 & 0 & 0 & 0 \\
\hline 113 & 1795 & 0 & 0 & 1795 & 0 & 0 & 0 & 0 \\
\hline 114 & 1879 & 43 & 47 & 1832 & 4 & 0 & 4 & 9.30 \\
\hline 115 & 1953 & 0 & 0 & 1953 & 0 & 0 & 0 & 0 \\
\hline 116 & 2412 & 109 & 99 & 2293 & 1 & 9 & 10 & 9.17 \\
\hline 117 & 1535 & 0 & 0 & 1535 & 0 & 0 & 0 & 0 \\
\hline 119 & 1987 & 444 & 424 & 1523 & 4 & 16 & 20 & 4.50 \\
\hline 121 & 1863 & 1 & 0 & 1861 & 0 & 1 & 1 & 100 \\
\hline 122 & 2476 & 0 & 0 & 2476 & 0 & 0 & 0 & 0 \\
\hline 123 & 1518 & 3 & 3 & 1515 & 0 & 0 & 0 & 0 \\
\hline 124 & 1619 & 47 & 56 & 1563 & 9 & 0 & 9 & 19.15 \\
\hline 202 & 2136 & 19 & 17 & 2115 & 0 & 2 & 2 & 10.53 \\
\hline 205 & 2656 & 82 & 93 & 2563 & 8 & 3 & 11 & 13.41 \\
\hline 209 & 3005 & 1 & 2 & 3003 & 1 & 0 & 1 & 100 \\
\hline 210 & 2650 & 194 & 201 & 2449 & 5 & 2 & 7 & 3.61 \\
\hline 212 & 2748 & 0 & 1 & 2747 & 1 & 0 & 1 & 100 \\
\hline 213 & 3251 & 220 & 220 & 3031 & 0 & 0 & 0 & 0 \\
\hline 215 & 3363 & 164 & 164 & 3199 & 0 & 0 & 0 & 0 \\
\hline 217 & 2208 & 162 & 152 & 2036 & 3 & 7 & 10 & 6.17 \\
\hline 220 & 2048 & 0 & 0 & 2048 & 0 & 0 & 0 & 0 \\
\hline 221 & 2427 & 396 & 403 & 2024 & 6 & 1 & 7 & 1.77 \\
\hline 223 & 2605 & 473 & 473 & 2132 & 0 & 0 & 0 & 0 \\
\hline 228 & 2053 & 362 & 362 & 1691 & 0 & 0 & 0 & 0 \\
\hline 230 & 2256 & 1 & 1 & 2255 & 0 & 0 & 0 & 0 \\
\hline 231 & 1573 & 2 & 2 & 1571 & 0 & 0 & 0 & 0 \\
\hline 234 & 2753 & 3 & 3 & 2750 & 0 & 0 & 0 & 0 \\
\hline 36 & 80872 & 3408 & 5804 & 77366 & 50 & 48 & 98 & 2.87 \\
\hline
\end{tabular}


Table 5. PVC detection speed performance comparison on the following mother wavelets: db2, db4, coif6 and sym4. It was taken into account a half-hour record from MIT-BIH Arrhythmia Database. The processing time was verified in seconds.

\begin{tabular}{cc}
\hline Mother Wavelet & Speed (s) \\
\hline $\mathrm{db} 2$ & 61.2 \\
$\mathrm{db} 4$ & 61.8 \\
$\mathrm{coif6}$ & 62.3 \\
$\mathrm{sym} 4$ & 61.8 \\
\hline
\end{tabular}

\section{Discussions}

ECG analysis is an important issue related to healthcare, especially for telecardioloy applications. In this paper we presented a system based on Redundant Discrete Wavelet Transform (RDWT) associated with adaptive threshold methods for Premature Ventricular Contraction (PVC) detection with real-time approach.

There are several works that use the Wavelet Transform for ECG analysis, and the Wavelet family most used is the Daubechies family, especially the mother Wavelet db4 (Arrais et al., 2016; Sasikala and Wahidabanu, 2010; Singh and Tiwari, 2006). However, is not sure the choice for $\mathrm{db} 4$. In this paper was presented a comparative study between three Wavelet families and some of their mother wavelets: Daubechies ( db2, db4, db6, db8 and $\mathrm{db} 12$ ), Coiflets (coif6, coif12 and coif18) and Symlets (sym4, sym6, sym8 and sym10). The Table 2 showed the comparative results.

The comparative study was based on standard deviation analysis between the ECG original signal energy and the scaling coefficients energy at all resolution level. It was analyzed around thirty recordings from MIT-BIH Arrhythmia Database as reference, which presents both ECG signal with and without PVC occurrence. The results showed that the low-order filters provide the best results for ECG analysis. This is a very interesting result, since the numerical processing time is directly proportional to the order filter, so low-order filters require less computational costs time.

It was verified that the best mother wavelet for ECG signal analysis is the $\mathrm{db} 2$ (Daubechies family. Furthermore, it can be observed that the Daubechies family provides the best results if compared to Coiflets wavelet family. However, comparing the Daubechies and Symlets family the results were very close, what was expected, since Symlets family is a modified version of Daubechies wavelets with increased symmetry. However, the Symlets family mathematical implementation is more complex if compared to Daubechies family.

It was observed that based on the energy of the wavelet coefficients and threshold methods can detect the occurrence of a premature ventricular contraction. Besides that, the energy wavelet coefficients provide the frequency band where the PVC energy present the most portion and the PVC duration time influence in the heartbeat. Thus, the frequency band can be used as indicative parameter of the ectopic stimulus origin (Malmivuo and Plonsey, 1995).

The database chosen for validated the algorithm is one of the most used for this propose. The Sensitivity $(\mathrm{Se})$, Positive Predictivity $\left(P^{+}\right)$and Specificity $(S p)$ are the essentially parameters should be used to evaluate the performance results of arrhythmia detection algorithms (Kohler et al., 2002; Martinez et al., 2004; Wang, 2013). The Sensitivity $(\mathrm{Se})$ indicates the algorithm's ability to detect truly positive characteristics, therefore high Sensitivity indicates few false negative cases. The Positive Predictivity Value $\left(P^{+}\right)$indicates the algorithm's ability to detect positive characteristics that are really positive, so the lower number of false positives provides the better algorithm positive predictability. The Specificity $(S p)$ indicates the algorithm's ability to detect negative characteristics, therefore high Specificity indicates few false positive cases.

In general, Sensitivity and Specificity are the most indicated parameters for overall accuracy algorithm, whereas Sensitivity and Positive Predictivity Values are the indicated for algorithm performance measures (Wang, 2013). Therefore, based on these results it was found that there is a trade-off between the choice of wavelet filter order and the performance parameters. The high order wavelet filter presents a higher Sensitivity and Specificity if compared to low order filter, as verified between $\mathrm{db} 2$ and $\mathrm{db} 4$. Whereas the Positive Predictivity Value presents a high value for low order filter and low value for high order wavelet filter.

Although the $\mathrm{db} 2$ and $\mathrm{db} 4$ performance have been very close (Table 3 ), it was chosen the $\mathrm{db} 2$ for proposed system, in order to achieve the best trade-off, with good Sensitivity, Positive Predictivity Value, Specificity and low order wavelet filter implementation algorithm. Through the Table 5 can verified that $\mathrm{db} 2$ presents the best speed performance. So, the $\mathrm{db} 2$ mother wavelet is the most indicated mother wavelet that provides reliable and accurate detection of the ECG signal characteristic.

\section{Acknowledgements}

The authors would like to thank the Brazilian National Research Council (CNPq), the Federal University of Rio Grande do Norte and the Rural Federal University of Semi-Arid for the support throughout the work. 


\section{References}

Andrade L, Leao MTP. Fault location for transmission lines using wavelet. IEEE Latin Am Trans. 2014; 12(6):1043-8. http://dx.doi.org/10.1109/TLA.2014.6893998.

Arrais E Jr, Valentim RAM, Brandão GB. Real time QRS detection based on redundant discrete wavelet transform. IEEE Latin Am Trans. 2016; 14(4):1662-8. http://dx.doi.org/10.1109/ TLA.2016.7483498.

Arrais E, Roda VO, Sousa CM No, Ribeiro RLA, Costa FB. FPGA versus DSP for wavelet transform based voltage sags detection. In: Proceedings 2014 IEEE International Instrumentation and Measurement Technology Conference (I2MTC); 2014 May 12-15; Montevideo, Uruguay. USA: IEEE; 2014. p. 643-7. http://dx.doi.org/10.1109/I2MTC.2014.6860822.

Bortolan G, Degani R, Willems JL. ECG classification with neural networks and cluster analysis. Proc in Comp in Cardio. 1991; 1991:177-80.

Braunwald E, Antman EM, Beasley JW, Califf RM, Cheitlin MD, Hochman JS, Jones RH, Kereiakes D, Kupersmith J, Levin TN, Pepine CJ, Schaeffer JW, Smith EE 3rd, Steward DE, Theroux P, Gibbons RJ, Antman EM, Alpert JS, Faxon DP, Fuster V, Gregoratos G, Hiratzka LF, Jacobs AK, Smith SC Jr. ACC/ AHA 2002 guideline update for the management of patients with unstable angina and non-st-segment elevation myocardial infarction: summary article. A report of the American College of Cardiology. J Am Coll Cardiol. 2002; 40(7):1366-74. http:// dx.doi.org/10.1016/S0735-1097(02)02336-7. PMid:12383588.

Burrus CS, Gopinath RA, Guo H. Introduction to wavelets and wavelet transforms: a primer. New Jersey: Prentice Hall; 1997.

Chazal P, O’Dwyer M, Reilly RB. Automatic classification of heartbeats using ecg morphology and heartbeat interval features. IEEE Trans Biomed Eng. 2004; 51(7):1196-206. http://dx.doi. org/10.1109/TBME.2004.827359. PMid:15248536.

Chazal P, Reilly RB. A patient-adapting heartbeat classifier using ecg morphology and heartbeat interval features. IEEE Trans Biomed Eng. 2006; 53(12 Pt 1):2535-43. http://dx.doi. org/10.1109/TBME.2006.883802. PMid:17153211.

Chong JW, Esa N, McManus DD, Chon KH. Arrhythmia discrimination using a smart phone. IEEE J Biol Health Info. 2015; 19(3):815-24. PMid:25838530.

Clifford GD, Azuaje F, McSharry PE, editors. Advanced methods and tools for ECG analysis. Vol. 1. Norwood: Artech House; 2006. (Series Engineering in Medicine and Biology).

Costa FB, Driesen J. Assessment of voltage sag indices based on scaling and wavelet coefficient energy analysis. IEEE Trans Power Deliv. 2013; 28(1):336-46. http://dx.doi.org/10.1109/ TPWRD.2012.2218626.

Costa FB. Boundary wavelet coefficients for real-time detection of transients induced by faults and power-quality disturbances. IEEE Trans Power Deliv. 2014; 29(6):2674-87. http://dx.doi. org/10.1109/TPWRD.2014.2321178.

Dalvi RF, Zago GT, Andreao RV. Heartbeat classification system based on neural networks and dimensionality reduction. Res Biomed Eng. 2016; 32(4):318-26. http://dx.doi. org/10.1590/2446-4740.05815.
Daubechies I. Ten lectures on wavelets. Canada: Siam; 1992. (CBMS-NSF Regional Conference Series in Applied Mathematics). http://dx.doi.org/10.1137/1.9781611970104.

Di Marco LY, Chiari L. A wavelet-based ECG delineation algorithm for 32-bit integer online processing. Biomed Eng Online. 2011; 10(1):1-19. http://dx.doi.org/10.1186/1475925X-10-23. PMid:21457580.

Dotsinsky IA, Stoyanov TV. Ventricular beat detection in single channel electrocardiograms. Biomed Eng Online. 2004; 3(1):19. http://dx.doi.org/10.1186/1475-925X-3-3. PMid:14750981.

Gadêlha MFJ, Nicolosi DEC, França FFAC. Performance analysis of an interpretive electrocardiograph for use in a computer-aided diagnostic system for acute coronary syndromes. Rev Bras Eng Bioméd. 2012; 28(2):140-54. http://dx.doi. org/10.4322/rbeb.2012.019.

Gagnon MP, Duplantie J, Fortin JP, Landry R. Implementing telehealth to support medical practice in rural/remote regions: what are the conditions for success? Imp Sci. 2006; 1(1):1-8. http://dx.doi.org/10.1186/1748-5908-1-18. PMid:16930484.

Ge B, Ji KT, Ye HG, Li J, Li YC, Yin RP, Lin JF. Electrocardiogram features of premature ventricular contractions/ventricular tachycardia originating from the left ventricular outflow tract and the treatment outcome of radiofrequency catheter ablation. BMC Cardiovasc Disord. 2012; 12(1):1-11. http://dx.doi. org/10.1186/1471-2261-12-112. PMid:23186541.

Goldberger ALL, Amaral AN, Glass L, Hausdorff JM, Ivanov PC, Mark RG, et al. Physiobank, physiotoolkit, and physionet: Components of a new research resource for complex physiologic signals. Comp Cardio. 2000; 101(23):215-20. PMID: 10851218.

Grossmann A, Morlet J. Decomposition of hardy functions into square integrable wavelets of constant shape. SIAM J Math Anal. 1984; 15(4):723-36. http://dx.doi.org/10.1137/0515056.

Hamilton PS, Tompkins WT. Quantitative Investigation of QRS Detection Rules Using the MIT/BIH Arrhythmia Database. IEEE Trans Biomed Eng. 1986; 33(12):1157-65. http://dx.doi. org/10.1109/TBME.1986.325695. PMid:3817849.

Hersh WR, Helfand M, Wallace J, Kraemer D, Patterson P, Shapiro S, Greenlick M. Clinical outcomes resulting from telemedicine interventions: A systematic review. BMC Med Inform Decis Mak. 2001; 1(1):1-8. http://dx.doi.org/10.1186/14726947-1-5. PMid:11737882.

Hirose H, Ishikawa S, Gotoh T, Kabutoya T, Kayaba K, Kajii E. Cardiac mortality of premature ventricular complexes in healthy people in Japan. J Cardiol. 2010; 56(1):23-6. http:// dx.doi.org/10.1016/j.jjcc.2010.01.005. PMid:20350513.

$\mathrm{Hu}$ YH, Palreddy S, Tompkins WJ. A patient-adaptable ecg beat classifier using a mixture of experts approach. IEEE Trans Biomed Eng. 1997; 44(9):891-900. http://dx.doi. org/10.1109/10.623058. PMid:9282481.

Inan OT, Giovangrandi L, Kovacs GTA. Robust neural-networkbased classification of premature ventricular contractions using wavelet transform and timing interval features. IEEE Trans Biomed Eng. 2006; 53(12):2507-15.

Iwasa A, Hwa M, Hassankhani A, Liu T, Narayan SM. Abnormal heart rate turbulence predicts the initiation of ventricular 
arrhythmias. Pac Clin Electroph. 2005; 28(11):1189-97. http:// dx.doi.org/10.1111/j.1540-8159.2005.50186.x.

Jia L, Yue-Chun L, Kang-Ting J, Na-Dan Z, Jia-Xuan L, Wen-Wu Z, Peng-Lin Y, Ji-Fei T, Jia-Feng L. Premature ventricular contractions originating from the left ventricular septum: results of radiofrequency catheter ablation in twenty patients. BMC Cardio Disease. 2011; 11(1):1-8. http://dx.doi. org/10.1186/1471-2261-11-27. PMid:21635765.

Kim J, Min SD, Lee M. An arrhythmia classification algorithm using a dedicated wavelet adapted to different subjects. Biomed Eng Online. 2011; 10(1):1-19. http://dx.doi.org/10.1186/1475925X-10-56. PMid:21707989.

Kohler BU, Hennig C, Orglmeister R. The principles of software QRS detection. IEEE Eng Med Biol Mag. 2002; 21(1):42-57. http://dx.doi.org/10.1109/51.993193. PMid:11935987.

Lek-uthai A, Ittatirut S, Teeramongkonrasmee A. Algorithm development for real-time detection of premature ventricular contraction. In: TENCON 2014 - 2014 IEEE Region 10 Conference; 2014 Oct 22-25; Bangkok, Thailand. USA: IEEE; 2014. p. 1-5. http://dx.doi.org/10.1109/TENCON.2014.7022418.

Li C, Zheng C, Tai C. Detection of ECG characteristic points using wavelet transforms. IEEE Trans Biomed Eng. 1995; 42(1):21-8. http://dx.doi.org/10.1109/10.362922. PMid:7851927.

Lim JS. Finding features for real-time premature ventricular contraction detection using a fuzzy neural network system. IEEE Trans Neural Netw. 2009; 20(3):522-7. http://dx.doi. org/10.1109/TNN.2008.2012031. PMid:19179246.

Madeiro JPV, Cortez PC, Marques JAL. Performance comparison analysis of Wavelet and Hilbert transforms for QRS detection in ECG. Rev Bras Eng Bioméd. 2009; 25:153-66. http://dx.doi. org/10.4322/rbeb.2012.072.

Mallat SG. A theory for multiresolution signal decomposition: the wavelet representation. IEEE Trans Pattern Anal Mach Intell. 1989; 11(7):674-93. http://dx.doi.org/10.1109/34.192463.

Mallat SG. A wavelet tour of signal processing: the sparse way. USA: Academic Press; 2008.

Malmivuo J, Plonsey R. Bioelectromagnetism: principles and applications of bioelectric and biomagnetic fields. Oxford: Oxford University Press; 1995. http://dx.doi.org/10.1093/ac prof:oso/9780195058239.001.0001.

Mann DL, Zipes DP, Libby P, Bonow RO, editors. Braunwald's heart disease: a textbook of cardiovascular medicine. Philadelphia: Saunders Elsevier; 2014.

Martinez J, Almeida R, Olmos S, Rocha A, Laguna P. A wavelet-based ecg delineator: evaluation on standard databases. IEEE Trans Biomed Eng. 2004; 51(4):570-81. http://dx.doi. org/10.1109/TBME.2003.821031.

Martis RJ, Acharya UR, Adeli H. Current methods in electrocardiogram characterization. Comput Biol Med. 2014; 48:133-49. http://dx.doi.org/10.1016/j.compbiomed.2014.02.012. PMid:24681634.

Martis RJ, Acharya UR, Min LC. ECG beat classification using PCA, LDA, ICA and discrete wavelet transform. Biomed Signal Process Control. 2013; 8(5):437-48. http://dx.doi.org/10.1016/j. bspc.2013.01.005.
Moody G, Mark R. The impact of the mit-bih arrhythmia database. IEEE Eng Med Biol Mag. 2001; 20(3):45-50. http:// dx.doi.org/10.1109/51.932724. PMid:11446209.

Morris F, Brady WJ, Camm J. ABC of clinical electrocardiography. USA: John Wiley \& Sons; 2009.

Natale A. Handbook of cardiac electrophysiology. Boca Raton: CRC Press; 2007. http://dx.doi.org/10.3109/9780203089866.

Nazarahari M, Ghorbanpour Namin S, Davaie Markazi AH, Kabir Anaraki A. A multi-wavelet optimization approach using similarity measures for electrocardiogram signal classification. Biomed Sig Proc Cont. 2015; 20(400):142-51. http://dx.doi. org/10.1016/j.bspc.2015.04.010.

Nieminaki M, Ruha A, Kemppainen J, Nissila S, Myilyla R. Real-time detection of premature ventricular contraction using a matched filter bank. In: Proceedings of the First Joint BMES/ EMBS Conference. 1999 IEEE Engineering in Medicine and Biology 21st Annual Conference and the 1999 Annual Fall Meeting of the Biomedical Engineering Society; 1999 Oct 13-16; Atlanta, GA, USA. USA: IEEE; 1999. p. 269. http:// dx.doi.org/10.1109/IEMBS.1999.802338.

Orozco-Duque A, Martinez-Tabares FJ, Gallego J, Rodriguez CA, Mora ID, Castellanos-Dominguez G, et al. Classification of premature ventricular contraction based on discrete wavelet transform for real time applications. In: Health Care Exchanges (PAHCE) 2013 Pan American; 2013 Apr 29-May 4; Medellin, Colombia. USA: IEEE; 2013.p. 1-5. http://dx.doi.org/10.1109/ PAHCE.2013.6568330.

Pan J, Tompkins WJ. A real-time QRS detection algorithm. IEEE Trans Biomed Eng. 1985; 32(3):230-6. http://dx.doi. org/10.1109/TBME.1985.325532. PMid:3997178.

Pedireddi LB, Srinivasan B. Characterization of atmospheric turbulence effects and their mitigation using wavelet-based signal processing. IEEE Trans Commun. 2010; 58(6):1795802. http://dx.doi.org/10.1109/TCOMM.2010.06.090194.

Percival AT, Walden DB. Wavelet methods for time series analysis. New York: Cambridge University Press; 2000. http:// dx.doi.org/10.1017/CBO9780511841040.

Saleh SA, Ahshan R, Moloney CR. Wavelet-based signal processing method for detecting ice accretion on wind turbines. IEEE Trans Sust Ener. 2012; 3(3):585-97. http://dx.doi. org/10.1109/TSTE.2012.2194725.

Sasikala P, Wahidabanu R. Robust r peak and qrs detection in electrocardiogram using wavelet transform. Inter J of Advan Comp Sci and App-IJACSA. 2010; 1(6):48-53.

Sayadi O, Shamsollahi MB, Clifford GD. Robust detection of premature ventricular contractions using a wave-based Bayesian framework. IEEE Trans Biomed Eng. 2010; 57(2):353-62. http:// dx.doi.org/10.1109/TBME.2009.2031243. PMid:19758851.

Shen Z, Hu C, Li P, Meng MQH. Research on premature ventricular contraction real-time detection based support vector machine. In: 2011 IEEE International Conference on Information and Automation (ICIA); 2011 June 6-8; Shenzhen, China. USA: IEEE; 2011.p. 864-9. http://dx.doi.org/10.1109/ ICINFA.2011.5949116.

Shyu LY, Wu YH, Hu W. Using wavelet transform and fuzzy neural network for vpc detection from the holter ecg. IEEE Trans 
Biomed Eng. 2004; 51(7):1269-73. http://dx.doi.org/10.1109/ TBME.2004.824131. PMid:15248543.

Singh BN, Tiwari AK. Optimal selection of wavelet basis function applied to ECG signal denoising. Dig Sig Proc. 2006; 16(3):275-87. http://dx.doi.org/10.1016/j.dsp.2005.12.003.

Solosenko A, Petrenas A, Marozas V. Photoplethysmographybased method for automatic detection of premature ventricular contractions. IEEE Trans Biomed Circuits Syst. 2015; 9(5):662-9. http://dx.doi.org/10.1109/TBCAS.2015.2477437. PMid:26513800.

Thakor N, Webster J, Tompkins WJ. Estimation of QRS complex power spectra for design of a qrs filter. IEEE Trans Biomed Eng. 1984; 31(11):702-6. http://dx.doi.org/10.1109/ TBME.1984.325393. PMid:6500590.

Valentim RAM, Araújo BG, Guedes TAL. A telessaúde no Brasil e a inovação tecnológica na atenção primária. Natal: EDUFRN; 2015.

Wang J. Proposed new requirements for testing and reporting performance results of arrhythmia detection algorithms.
In: Computing in Cardiology; 2013 Sept 22-25; Zaragoza, Spain. USA: IEEE; 2013. p. 967-70.

World Health Organization. WHO methods and data sources for country-level causes of death 2000-2015 [Internet]. Geneva: WHO; 2017 [cited 2018 Feb 28]. Available from: http://www.who.int/healthinfo/global_burden_disease/ GlobalCOD_method_2000_2015.pdf?ua=1

Yochum M, Renaud C, Jacquir S. Automatic detection of p, qrs and $t$ patterns in 12 leads ECG signal based on CWT. Biomed Sig Proc Contr. 2016; 25:46-52. http://dx.doi.org/10.1016/j. bspc.2015.10.011.

Zago GT, Andreão RV, Rodrigues SL, Mill JG, Sarcinelli M Fo. ECG-based detection of left ventricle hypertrophy. Res Biomed Eng. 2015; 31(2):125-32. http://dx.doi.org/10.1590/24464740.0691

Zheng C, Li J, Lin JX, Wang LP, Lin JF. Where is the exact origin of narrow premature ventricular contractions manifesting qr in inferior wall leads? BMC Cardiol Diseas. 2016; 16(64):1-12. http://dx.doi.org/10.1186/s12872-016-0240-4. PMid:27044385. 